\title{
The effect of steam sterilization on the accuracy of spring-style mechanical torque devices for dental implants
}

This article was published in the following Dove Press journal:

Clinical, Cosmetic and Investigational Dentistry

30 July 2012

Number of times this article has been viewed

\author{
Minoo Mahshid' \\ Aboulfazl Saboury' \\ Ali Fayaz' \\ Seyed Jalil Sadr' \\ Friedrich Lampert ${ }^{2}$ \\ Maziar Mir ${ }^{2,3}$ \\ 'Department of Prosthodontics, \\ Dental School, Shahid Beheshti \\ University of Medical Sciences, \\ Tehran, Iran; ${ }^{2}$ Department of \\ Conservative Dentistry, Aachen RWTH \\ Hospital, Aachen, Germany; ${ }^{3}$ Beckman \\ Laser Institute, UCI, Irvine, CA, USA
}

Correspondence: Aboulfazl Saboury Aachen RWTH Hospital, Pauwelsstr 30 52074, Aachen, Germany Emailmmir@ukaachen.de
Background: Mechanical torque devices (MTDs) are one of the most commonly recommended devices used to deliver optimal torque to the screw of dental implants. Recently, high variability has been reported about the accuracy of spring-style mechanical torque devices (S-S MTDs). Joint stability and survival rate of fixed implant supported prosthesis depends on the accuracy of these devices. Currently, there is limited information on the steam sterilization influence on the accuracy of MTDs. The purpose of this study was to assess the effect of steam sterilization on the accuracy ( $\pm 10 \%$ of the target torque) of spring-style mechanical torque devices for dental implants.

Materials and methods: Fifteen new S-S MTDs and their appropriate drivers from three different manufacturers (Nobel Biocare, Straumann [ITI], and Biomet 3i [3i]) were selected. Peak torque of devices ( 5 in each subgroup) was measured before and after autoclaving using a Tohnichi torque gauge. Descriptive statistical analysis was used and a repeated-measures ANOVA with type of device as a between-subject comparison was performed to assess the difference in accuracy among the three groups of spring-style mechanical torque devices after sterilization. A Bonferroni post hoc test was used to assess pairwise comparisons.

Results: Before steam sterilization, all the tested devices stayed within $10 \%$ of their target values. After 100 sterilization cycles, results didn't show any significant difference between raw and absolute error values in the Nobel Biocare and ITI devices; however the results demonstrated an increase of error values in the 3 i group $(P<0.05)$. Raw error values increased with a predictable pattern in 3 i devices and showed more than a $10 \%$ difference from target torque values (maximum difference of $14 \%$ from target torque was seen in $17 \%$ of peak torque measurements).

Conclusion: Within the limitation of this study, steam sterilization did not affect the accuracy ( $\pm 10 \%$ of the target torque) of the Nobel Biocare and ITI MTDs. Raw error values increased with a predictable pattern in $3 i$ devices and showed more than $10 \%$ difference from target torque values. Before expanding upon the clinical implications, the controlled and combined effect of aging (frequency of use) and steam sterilization needs more investigation.

Keywords: accuracy, steam sterilization, mechanical torque devices, spring-style, dental implants

\section{Introduction}

Torque is a convenient, measurable means of developing desired tension within a screw. Applied torque develops a force within the screw called preload. ${ }^{1}$ The elastic recovery of the screw after elongation creates the clamping force that pulls the prosthesis and the implant together. ${ }^{2}$ Clamping force is usually proportionate to tightening 
torque. ${ }^{3}$ Dental implant screw joints tightened to lower preload values cannot achieve the mechanical integration in implant abutment interface. ${ }^{4,5}$

Mechanical torque devices (MTDs) are one of the most commonly recommended devices used to deliver optimal torque to the screw. Two types of mechanical torque devices are common in clinical use. These two types are toggle-type or friction-style and beam-type or spring-style. Current literature supports the view that spring-style mechanical torque devices (S-S MTDs) are more accurate than frictionstyle ones. ${ }^{6}$ The literature offers little information on the possible influence of steam sterilization on the accuracy of S-S MTDs. Cehreli et al examined the accuracy of new and used S-S MTDs, and their results showed a slight decrease in delivered torque output as a consequence of clinical use. ${ }^{7}$ McCracken et al also assessed the accuracy of S-S MTDs in clinical service, and found that these devices have the capability of producing accurate torque values within $10 \%$ of their target torque value. ${ }^{8}$ Recently, it was demonstrated that several of the tested S-S MTDs were not accurate and produced misleading values. ${ }^{9}$

Due to the high inaccuracy rate reported among S-S MTDs, and given that the mechanisms for these effects are unknown, this study aimed to investigate the effect of steam sterilization on the accuracy ( $\pm 10 \%$ of the target torque) of S-S MTDs.

The null hypothesis was that there would be no significant difference in the accuracy of S-S MTDs after 100 autoclave cycles of steam sterilization.

\section{Materials and methods}

Fifteen new S-S MTDs from three different implant manufacturers were evaluated to determine the effect of steam sterilization on their accuracy (within 10\% of the target value). Five samples from each of the three types of selected spring-style mechanical torque devices were tested, from the following manufacturers: (1) Nobel Biocare (Goteborg, Sweden); (2) Straumann (ITI), (Institut Straumann, Basel, Switzerland); and (3) Biomet 3i (3i) (Palm Beach Gardens, FL).

Target torque was $35 \mathrm{Ncm}$ for all the tested devices. Total specimen size of fifteen devices was selected according to other studies ${ }^{5}$ and considering the effect size of $0.37 \mathrm{Ncm}$, standard deviation $(\mathrm{SD})=0.13$ and $\mathrm{SD}=0.1$ using a two-level factorial design. Each manufacturer was given an abbreviation, and each device was randomly assigned a number from 11 to 15 in each group and then labeled accordingly: Nobel Biocare (X11 to X15), ITI (Y11 to Y15), 3i (Z11 to Z15). The sequence for testing the device was also randomized.

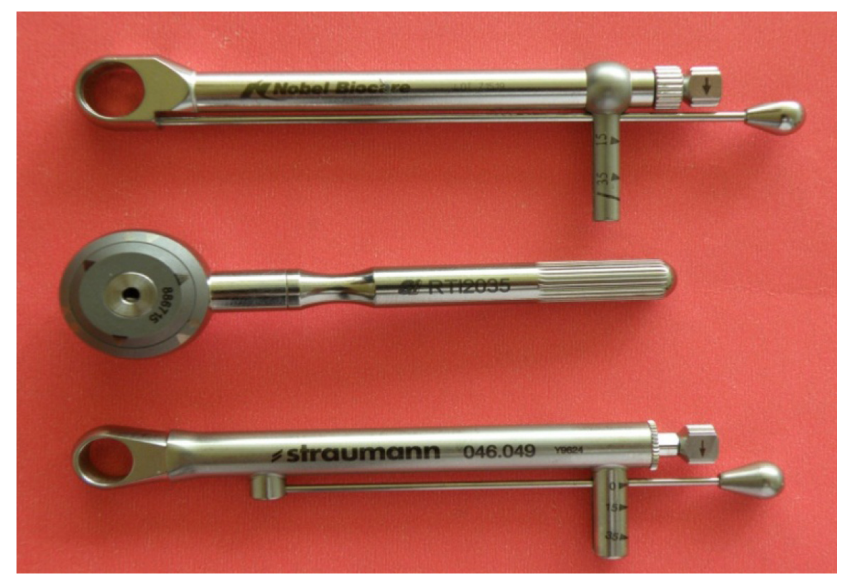

Figure I Spring-style mechanical torque devices tested.

Note: Top, Nobel Biocare; (Goteborg, Sweden); middle, Biomet 3i (Palm Beach Gardens, FL); bottom, Straumann (ITI) (Institut Straumann, Basel, Switzerland).

The Tohnichi torque gauge (6Tohnichi-BTG (-S); Tohnichi Mfg. Co, Ltd, Tokyo, Japan) was used to measure the peak torque values (actual torque output at a predetermined target torque) of each device. The torque gauge was new and calibrated by the manufacturer to be accurate within $\pm 2 \%$ of the full scale.

Appropriate drivers for each device were selected. Drivers were clamped in three-jaw chuck of torque gauge (Figure 2). The torque gauge was fixed in a vice for stability (Figure 3).

After connecting the devices to the driver, the torque indicator on the gauge was set to zero. Each device was tested by applying the torque slowly over 4 seconds. ${ }^{8}$ Force was applied to the S-S MTDs until the beam was flexed to the point

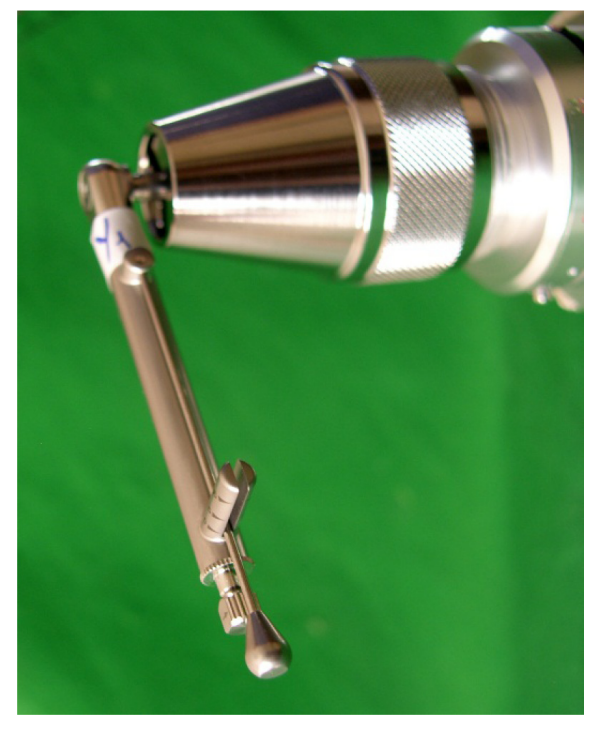

Figure 2 Drivers for each of the S-S MTDs were clamped in a three-jaw chuck of a torque gauge.

Abbreviation: S-S MTDs, spring-style mechanical torque devices. 


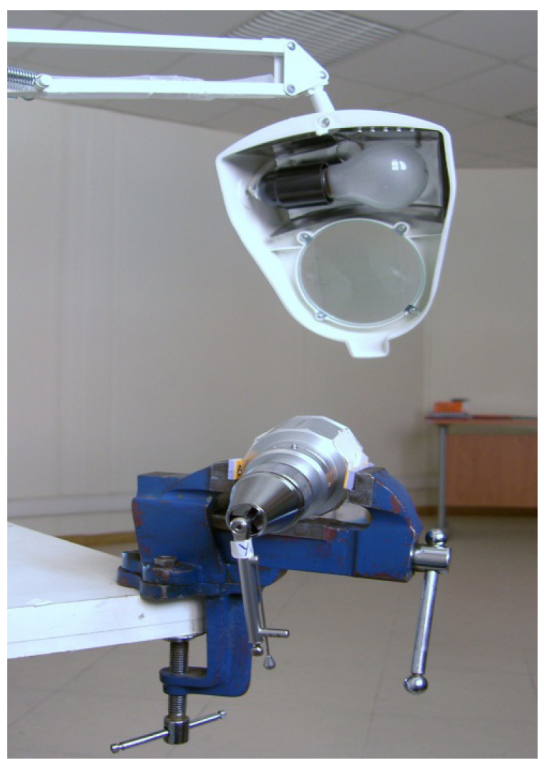

Figure 3 The torque gauge was fixed in a vice for stability and aligned with a magnifier.

that the desired torque was achieved visually at a precalibrated target torque value for the ITI and Nobel Biocare devices. For $3 \mathrm{i}$ devices alignment of triangular and diamond points demonstrated the application of the target values (Figure 4). The torque was applied by one operator that was blind to the measured values, and the other operator registered the peak torque values presented on the torque indicator of the gauge for each device using a magnifier (Figure 5).

The procedure of peak torque measurement was performed ten times before steam sterilization. Then preparation steps before sterilization were considered for each group of mechanical torque devices as proposed by the manufacturers. Nobel Biocare devices (X11-X15) should be dismantled for disinfection, cleaning and drying and then the parts should be assembled before sterilization (Figure 6). In the ITI group (Y11-Y15), dismantling of devices is proposed. Each component should be disinfected, cleaned, and dried, but sterilized separately (Figure 7). For 3i samples (Z11-Z15), dismantling of the device is not proposed and disinfection of the outer surface is the only protocol to be considered (Figure 8). Figure 9 demonstrates the error bar and confidence interval of mean raw error compared to target torque for three groups of (S-S MTDs), before and after steam sterilization.

In order to simulate the contamination of these devices with saliva during surgical and prosthetic procedures, devices were immersed in artificial saliva (BioXtra; Solarfarma, Knokke, Belgium) and then disinfected for 15 minutes with the $2 \%$ phenols and aldehyde-free, non-fixing disinfectant (Deconex 53
A

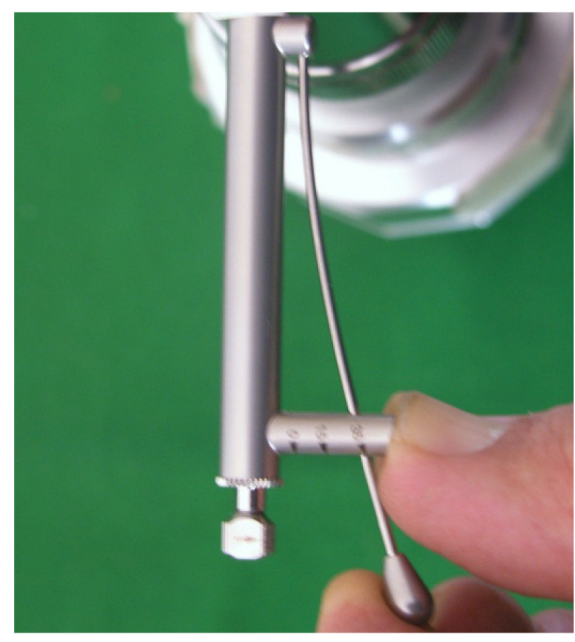

B

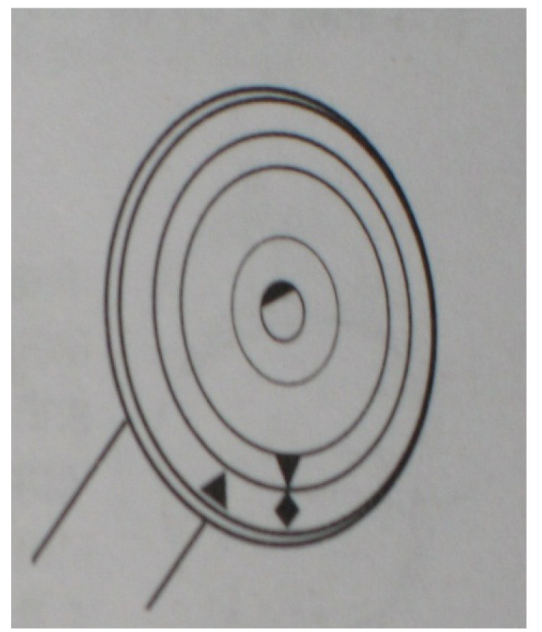

Figure 4 Alignment of the S-S MTDs. (A) For Straumann (ITI) (Institut Straumann, Basel, Switzerland) and Nobel Biocare (Goteborg, Sweden) devices, force was applied to the S-S MTDs until flexing the beam at a precalibrated target torque could be achieved visually. (B) For Biomet $3 \mathrm{i}$ devices (Palm Beach Gardens, FL), alignment of triangular and diamond points demonstrated the application of target torque values. Abbreviation: S-S MTDs, spring-style mechanical torque devices.

plus; Borer Chemie AG, Zuchwil, Switzerland) before each autoclaving cycle. Devices were packed without touching the components, and were then put through the steam autoclave (Europa BXP; Tecno-Gaz S.p.A, Parma, Italy) cycle. This procedure was repeated 100 times. Sterilization occurred at $134^{\circ} \mathrm{C}$, with 0.9 bar vacuum pressure and 18 minutes of sterilization time. After 100 complete autoclave sterilization cycles, the devices were retested by 10 consecutive measurements at peak torque values. Absolute differences (raw error values), mean, and range of difference between the measured torque value and the target torque were evaluated before and after autoclaving. Descriptive statistical analysis was used and a repeated-measures analysis of variance was conducted with type of device acting as a between-subjects comparison. 


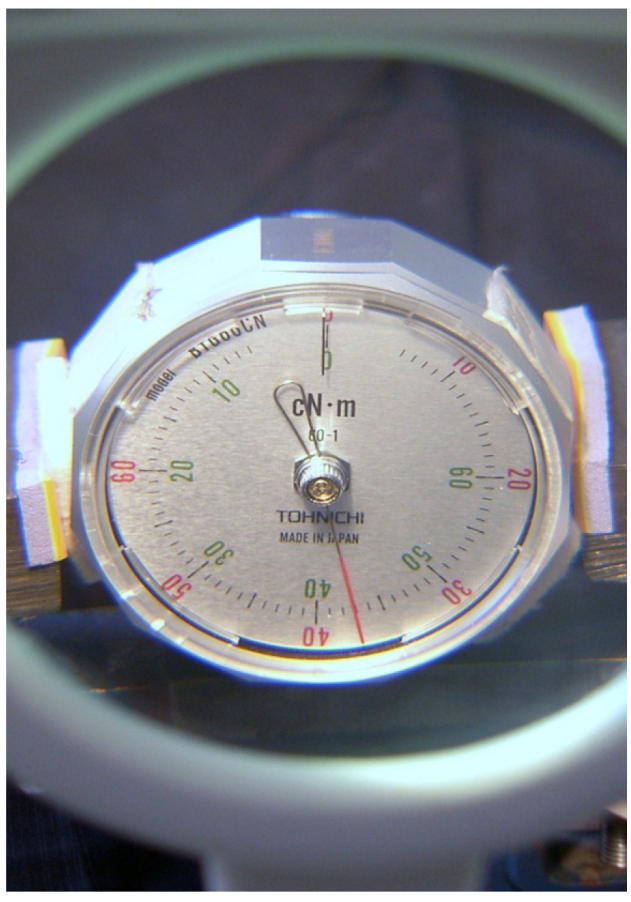

Figure 5 Peak torque values of each of the S-S MTDs presented on a torque indicator of the gauge. For each device, peak torque was registered using magnifier. Abbreviation: S-S MTDs, spring-style mechanical torque devices.

This was done to assess the difference in accuracy (within $10 \%$ of the target torque) among the three groups of S-S MTDs with a significance level of $P<0.05$. Bonferroni post hoc tests were used for pairwise comparisons.

\section{Results}

Descriptive values of mean, SD, minimum and maximum differences between the measured torque, and the targeted torque values for each group of S-S MTDs before and after steam sterilization are summarized in Table 1.

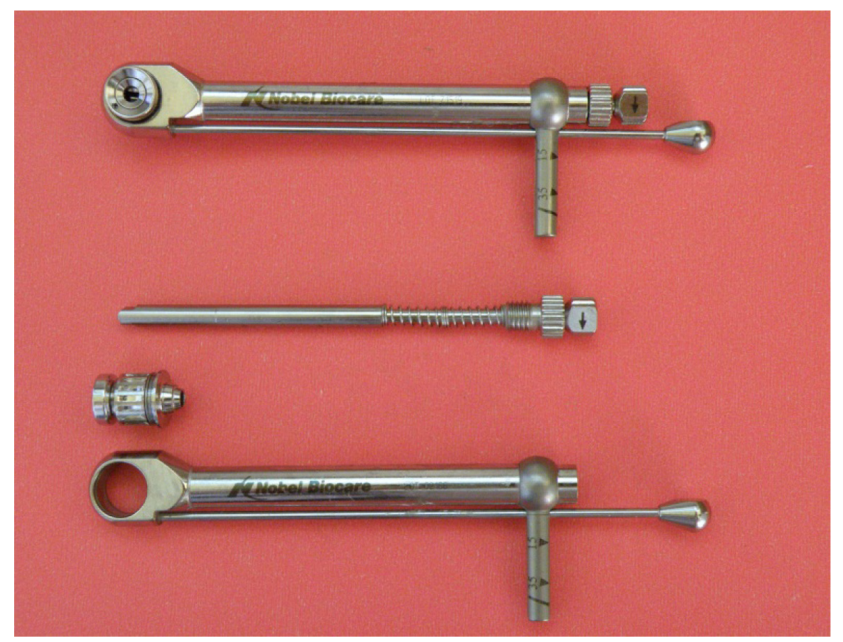

Figure 6 Nobel Biocare (Goteborg, Sweden) mechanical torque device.

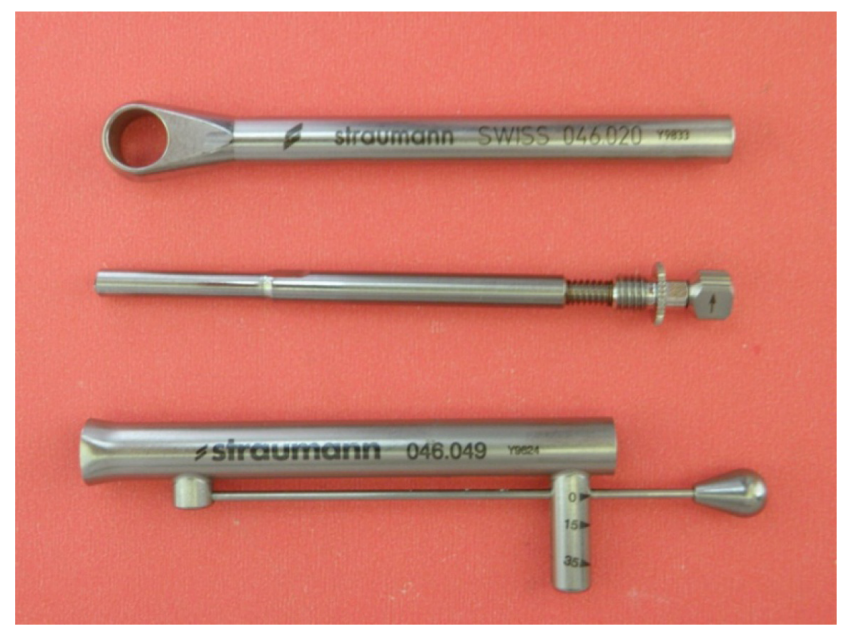

Figure 7 Straumann (ITI) (Institut Straumann, Basel, Switzerland) mechanical torque device.

\section{Discussion}

The data supports rejection of the null hypothesis as there was a statistically significant difference of error values after steam sterilization among 3 i devices $(P<0.05)$. Autoclaving (ie, sterilization in saturated steam under pressure) is considered to be the most reliable method known to destroy all forms of microbial life; however, instruments often corrode during autoclaving due to the hot steam medium. ${ }^{10,11}$ The literature offers little information on the possible influence of steam sterilization on the accuracy of S-S MTDs. Accuracy of MTDs is essential in producing consistent and appropriate preload on dental implant screws, thereby preventing the screw loosening. After an observation period of at least 5 years, screw loosening has been stated as the most common technical complication in implant dental restorations including implant-supported fixed dental prosthesis, both with ${ }^{12,13}$ or without ${ }^{14}$ cantilever extensions, and in implant-supported crowns..$^{15,16}$

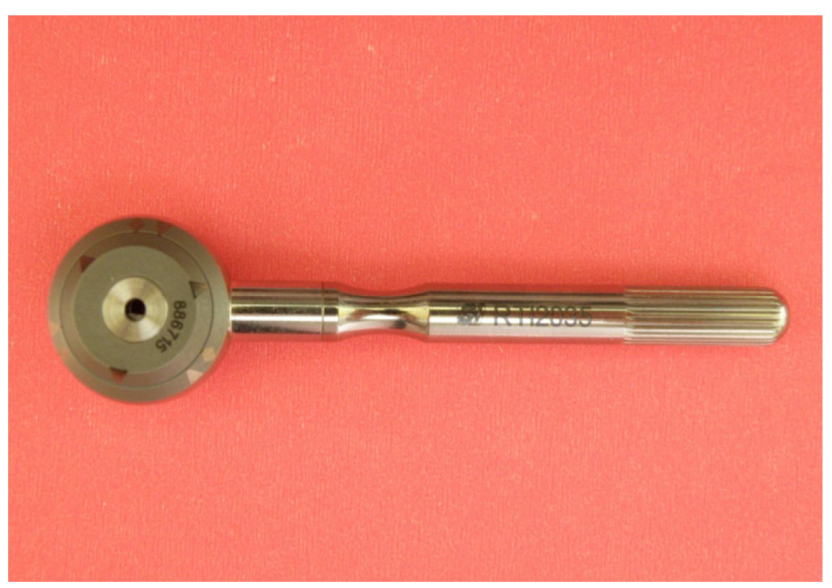

Figure 8 Biomet 3i (Palm Beach Gardens, FL) mechanical torque device. 


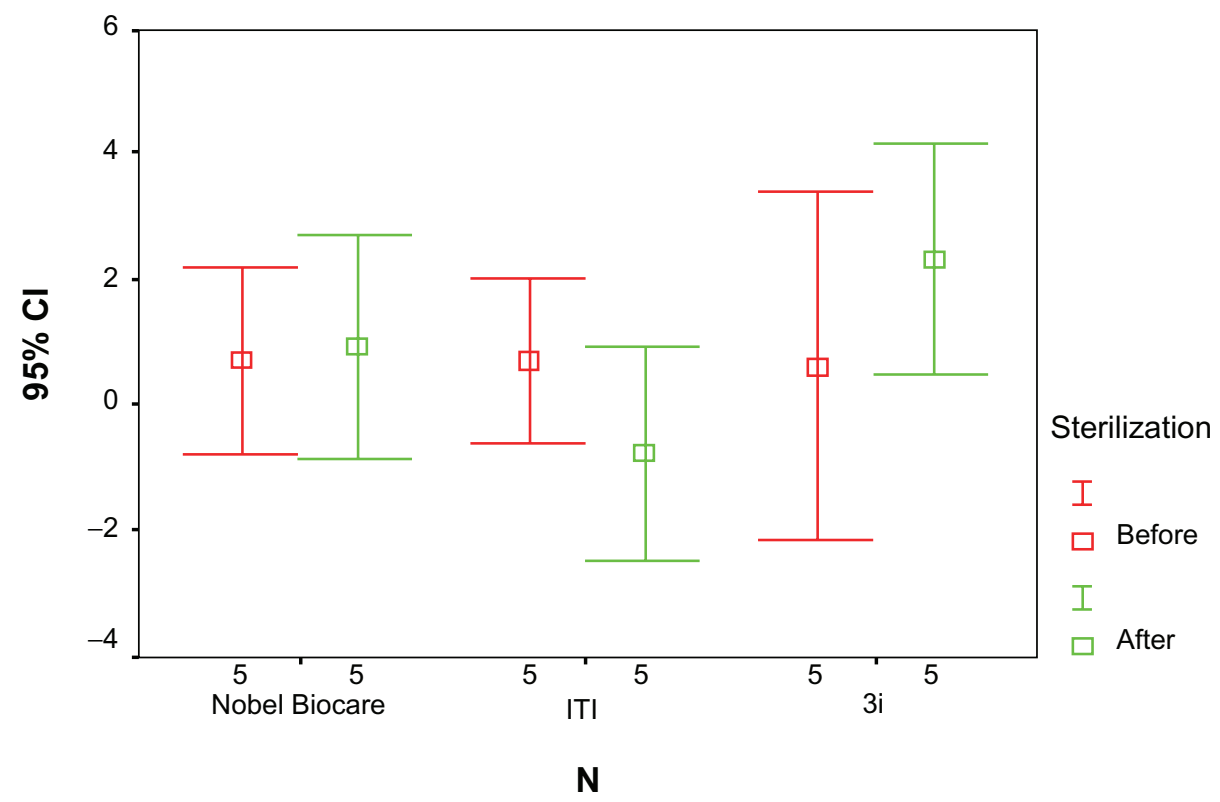

Figure 9 Comparison of raw error for the three S-S MTDs.

Notes: Error bar and $95 \% \mathrm{Cl}$ of mean raw error compared to target torque for three groups of spring-style mechanical torque devices before and after steam sterilization. Zero-level shows the target torque for each group (Nobel Biocare, Straumann [ITI], Biomet 3i [3i]).

Abbreviations: S-S MTDs, spring-style mechanical torque devices; $\mathrm{Cl}$, confidence interval; $\mathrm{N}$, number of tested devices.

Dismantling and assembling the parts before autoclaving for both the Nobel Biocare and ITI devices was proposed by manufacturers as preparatory steps. Low variability and range of values were seen in these two groups after steam sterilization cycles. This finding supports the results of previous studies, ${ }^{7,8}$ but sterilization protocols have not been clearly outlined in these studies. However, Santos et al demonstrated a level of inaccuracy in several of the tested S-S MTDs (Biomet 3i, Nobel Biocare, Straumann, and Conexao). ${ }^{9}$ For $20 \mathrm{Ncm}$ target torque values, $62.5 \%$ of measured values were accurate (within $10 \%$ of the target value); however, for $32 \mathrm{Ncm}$ target torque, only $33.3 \%$ of all values from each manufacturer were considered accurate. ITI torque devices showed the most consistent output of torque for both target torques (20 and $32 \mathrm{Ncm}$ ). All the devices had been in use for less than 2 years, but frequency of clinical use and sterilization protocols have not been identified in this study. When comparing the results of these studies with the results of the current study, these discrepant findings can be related to the careful and meticulous routine steps for preparation of the devices before autoclaving (dismantling, cleaning) in our study. It should be noted that aging of the devices has not been considered in the current study. The effect of aging on the accuracy

Table I Mean, SD, and range of difference between peak torque and target values before and after 100 steam sterilization cycles for S-S MTDs

\begin{tabular}{|c|c|c|c|c|c|}
\hline $\begin{array}{l}\text { Mechanical torque } \\
\text { devices of implant } \\
\text { manufacturers }\end{array}$ & $\begin{array}{l}\text { Measurement } \\
\text { time }\end{array}$ & $\begin{array}{l}\text { Absolute } \\
\text { difference }\end{array}$ & Minimum & Maximum & $\begin{array}{l}\text { Mean } \\
\text { difference } \pm \text { SD }\end{array}$ \\
\hline \multirow[t]{2}{*}{ Nobel Biocare } & Before & 1.28 & -1 & 3 & $0.9 \pm 0.89$ \\
\hline & After & 0.94 & -0.5 & 2 & $0.9 \pm 1.14$ \\
\hline \multirow[t]{2}{*}{ Straumann (ITI) } & Before & 1.14 & -2 & 1 & $0.7 \pm 0.67$ \\
\hline & After & 1.29 & -3 & 1.5 & $-0.8 \pm 1.09$ \\
\hline \multirow[t]{2}{*}{ Biomet 3i (3i) } & Before & 1.17 & -2.5 & 2.5 & $0.6 \pm 1.9$ \\
\hline & After & 2.3 & 0 & 5 & $2.3 \pm 1.2$ \\
\hline
\end{tabular}

Notes: Before sterilization, all the tested S-S MTDs stayed within 10\% of their target values. Steam sterilization did not affect the accuracy of Nobel Biocare and ITI mechanical torque devices. Range of difference of Nobel Biocare devices was -0.5 to $2 \mathrm{Ncm}$, and ITI devices demonstrated the range of difference of -3 to I.5 Ncm. In $3 \mathrm{i}$ mechanical torque devices, peak torque values showed significant differences after 100 autoclave cycles $(P<0.05)$. Absolute difference (raw error values) increased with predictable patterns in $3 \mathrm{i}$ devices and showed more than $10 \%$ difference from the target torque. Range of difference was 0 to $5 \mathrm{Ncm}$ for these devices. Abbreviations: SD, standard deviation; S-S MTDs, spring-style mechanical torque devices; $P$, probability level. 
of S-S MTDs is not clear, but the lifespan of these devices appears to be up to 700 times of use in clinical settings between 18 months and 7 years. This guideline appears to be most accurate and presents with low standard deviation $( \pm 1 \mathrm{Ncm}) .{ }^{8}$ High consistency and no operator-dependent effects on the fatigue behavior (up to 1000 times) of these devices was reported. These S-S MTDs showed a slight decrease in delivered torque output as a consequence of clinical use (1.5 Ncm lower than new devices). ${ }^{7}$ Etiology of the changes in torque output can include different sterilization protocols, differences between tested devices, and operator-dependent effects. ${ }^{7-9}$ The total combined effects of aging and sterilization on delivered torque output needs further investigation and clarification.

Congelation of lubricant inside the frictional-style devices was stated as a probable cause of inaccuracy in torque output; ${ }^{8}$ however, lubrication protocols have not been addressed or proposed in the outlined preparation steps before autoclaving S-S MTDs.

For the purpose of this study, torque values within $10 \%$ of the target torque was defined as the level of accuracy of tested devices. ${ }^{8,17}$ Accuracy within $10 \%$ of the target value of Nobel Biocare and ITI MTDs was not affected by autoclaving in the current study. In $3 \mathrm{i}$ MTDs, raw error values increased with predictable pattern in $17 \%$ of peak torque value measurements and showed a maximum difference of $14 \%$ from the target torque. Dismantling of components has not been proposed for $3 \mathrm{i}$ torque devices. Corrosion of the spring in the handle of the torque wrench was found to result in inaccurate torque delivery and limited torque output. ${ }^{10}$ Moreover, it is possible that dismantling prior to steam sterilization might result in the corrosion of the components in spring-style devices; however, this has not yet been evaluated. Each of these elements should be examined to determine whether these factors influence the accuracy of these devices.

The S-S MTDs used in the current in vitro study were new and had not been exposed to clinical procedures. Some studies use torque wrenches in clinical services to investigate their accuracy, ${ }^{7-10}$ but their results will not apply to every clinical situation given that too many confounding variables associated with sterilization, maintenance, and use of these devices exist, and these factors cannot be checked and defined precisely in these retrospective studies. Future observations may be needed to simulate clinical situations and control for confounding variables like aging of the devices. In considering the independent and combined effect of sterilization methods and aging, it will be possible to develop a clinical guideline to determine the sterilization protocols and calibration frequency needs of these devices.

\section{Conclusion}

Under the limitation of this in vitro study a number of conclusions were drawn. Steam sterilization did not affect the accuracy of ITI and Nobel Biocare MTDs. The majority of the new spring-style devices tested in this study delivered fairly consistent torque output within $10 \%$ of their preset target values after sterilization. Only the $3 i$ devices showed more than $10 \%$ difference from their target torque values (maximum of $14 \%$ ) in $17 \%$ of the measurements. Combined effects of sterilization and aging need to be evaluated before expanding and applying the clinical implications of the findings of this study.

\section{Acknowledgments}

This work has been sponsored by the Shahid Beheshti Research Department, and the authors would like to express their appreciation to Dr Mohammad Javad Kharazi Fard for statistical evaluation.

\section{Disclosure}

The authors report no conflicts of interest in this work.

\section{References}

1. McGlumphy EA, Mendel DA, Holloway JA. Implant screw mechanics. Dent Clin North Am. 1998;42(1):71-89.

2. Guda T, Ross TA, Lang LA, Millwater HR. Probabilistic analysis of preload in the abutment screw of a dental implant complex. J Prosthet Dent. 2008;100(3):183-193.

3. Burquete RL, Johns RB, King T, Patterson EA. Tightening characteristics for screwed joints in osseointegrated dental implants. $J$ Prosthet Dent. 1994;71(6):592-599.

4. Haack JE, Sakaguchi RL, Sun T, Coffey JP. Elongation and preload stress in dental implant abutment screws. Int J Maxillofac Implants. 1995;10(5):529-536.

5. Gratton DG, Aquilino SA, Stanford CM. Micromotion and dynamic fatigue properties of the dental implant-abutment interface. J Prosthet Dent. 2001;85(1):47-52.

6. Vallee MC, Conrad HJ, Basu S, Seong WJ. Accuracy of friction-style and spring-style mechanical torque limiting devices for dental implants. $J$ Prosthet Dent. 2008;100(2):86-92.

7. Cehreli MC, Akca K, Tonuk E. Accuracy of manual torque application device for morse-taper implants: a technical note. Int J Oral Maxillofac Implants. 2004;19(5):743-748.

8. McCracken MS, Mitchell L, Hegde R, Mavalli MD. Variability of mechanical torque-limiting devices in clinical service at a US dental school. J Prosthodont. 2010;19(1):20-24.

9. Santos GC Jr, Passos SP, Coelho Santos MJ. Accuracy of mechanical torque devices for implants used in Brazilian dental offices. Int $J$ Prosthodont. 2011;24(1):38-39.

10. Gutierrez J, Nicholls JI, Libman WJ, Butson TJ. Accuracy of the implant torque wrench following time in clinical service. Int J Prosthodont. 1997;10(6):562-567. 
11. Fajers CM, Holmlund LG, Stenman E. Corrosion during autoclave sterilization. II. Volatile organic amines as corrosion inhibitors. Acta Odontol Scand. 1968;26(1):23-34.

12. Zurdo J, Romao C, Wennstrom JL. Survival and complication rate of implant-supported fixed partial dentures with cantilevers: a systematic review. Clin Oral Implants Res. 2009;20(Suppl 4):59-66.

13. Aglietta M, Siciliano VI, Zwahlen M, et al. A systematic review of the survival and complication rates of implant supported fixed dental prostheses with cantilever extensions after an observation period of at least 5 years. Clin Oral Implants Res. 2009;20(5):441-451.

14. Kreissl ME, Gerds T, Muche R, Heydecke G, Strub JR. Technical complications of implant-supported fixed partial dentures in partially edentulous cases after an average observation period of 5 years. Clin Oral Implants Res. 2007;18(6):720-726.
15. Jung RE, Pjetursson BE, Glauser R, Zembic A, Zwahlen M, Lang NP. A systematic review of the 5-year survival and complication rates of implant-supported single crowns. Clin Oral Implants Res. 2008;19(2):119-130.

16. Pjetursson BE, Bragger U, Lang NP, Zwahlen MA. Comparison of survival and complication rates of tooth-supported fixed dental prosthesis (FPDs) and implant-supported FDPs and single crowns (SCs). Clin Oral implants Res. 2007;18(Suppl 3):97-113.

17. Standlee JP, Caputo AA, Chwu MY, Sun TT. Accuracy of mechanical torque-limiting devices for implants. Int J Oral Maxillofac Implants. 2002;17(2):220-224.

\section{Publish your work in this journal}

Clinical, Cosmetic and Investigational Dentistry is an international, peer-reviewed, open access, online journal focusing on the latest clinical and experimental research in dentistry with specific emphasis on cosmetic interventions. Innovative developments in dental materials, techniques and devices that improve outcomes and patient satisfac- tion and preference will be highlighted. The manuscript management system is completely online and includes a very quick and fair peerreview system, which is all easy to use. Visit http://www.dovepress. com/testimonials.php to read real quotes from published authors.

Submit your manuscript here: http://www.dovepress.com/clinical-cosmetic-and-investigational-dentistry-journal 